\title{
What determines the eyes' landing position in words?
}

\author{
STÉPHANIE DUCROT and JOËL PYNTE \\ CNRS and Université de Provence, Aix-en-Provence, France
}

\begin{abstract}
The place at which the eyes first fixate in a word during continuous reading, called the preferred landing position (PLP), is usually located halfway between the beginning and the middle of the word. To propose a mechanism that might account for the off-center location of the PLP, six eye movement experiments were conducted using a lexical decision task (Experiment 1) and a stimulus bisection task (Experiments 2-6). The type of stimulus-linguistic (words and nonwords) versus nonlinguistic (strings of hashes, dotted lines, and solid lines) — and the stimulus presentation side (left vs. right) were manipulated. The results showed that (1) stimulus discreteness versus continuousness is an important factor in saccade computation and (2) PLP asymmetry can be explained in terms of attentional and/or oculomotor processes.
\end{abstract}

The landing position in a word during continuous reading, originally called the preferred viewing location (Rayner, 1979) and which we will refer to as the preferred landing position (PLP), is usually located halfway between the beginning and the middle of the word (McConkie, Kerr, Reddix, \& Zola, 1988; Radach \& Kempe, 1993; Rayner, 1979; Vitu, O’Regan, \& Mittau, 1990). Explanations of this bias have been sought both in low-level oculomotor and/or perceptual constraints associated with saccade programming and execution and in high-level knowledgedependent positioning strategies. Regarding low-level sources of influence, it has been suggested, for instance, that readers might make an erroneous assessment of where the middle of the word actually is (see Coëffé \& O'Regan, 1987 , for a discussion of this idea). Alternatively, oculomotor noise could interfere with saccade computation and execution and lead to an aiming error (O'Regan \& LevySchoen, 1987; see also the notion of saccadic range error, McConkieet al., 1988). Moreover, when a sentence is being read, each word appears surrounded by other words, and it has been suggested that, under such circumstances, the initial fixation position is the weighted center of several words (Coëffé \& O'Regan, 1987; Findlay, 1982). According to this view, readers would aim at the word's center but would land to the left, owing to the properties of the oculomotor system.

Although perceptual and oculomotor factors of this kind are probably responsible for part of the PLP effect, they may not account for the whole phenomenon. For lan-

This paper was prepared while the first author was supported by a research fellowship from the Conseil Régional PACA. We thank Albrecht Inhoff, Alexander Pollatsek, and Keith Rayner for their helpful comments on previous versions of this paper. Correspondence should be addressed to S. Ducrot, Département de Psychologie Cognitive, Université de Provence, 29 avenue Robert Schuman, 13621 Aix-en-Provence cedex 1, France (e-mail: sducrot@up.univ-mrs.fr). guages such as English and French, most words can be guessed from their beginning (since word beginnings allegedly provide a higher degree of lexical constraint than do word endings; for a discussion, see Broerse \& Zwaan, 1966; Deutsch \& Rayner, 1999; Farid \& Grainger, 1996; O'Regan, Levy-Schoen, Pynte, \& Brugaillère, 1984), and it would not be surprising to find that readers tend to land where they know useful information is likely to be found. The PLP effect could also be seen as a side effect of parafoveal processing. In continuous reading, when a reader fixates word $n$, information is obtained parafoveally about word $n+1$, which facilitates its subsequent (foveal) processing (Henderson \& Ferreira, 1990; Morris, Rayner, \& Pollatsek, 1990; Rayner, 1975, 1986; Rayner, Well, Pollatsek, \& Bertera, 1982) and could have an impact on what landing position readers consider optimal (for a discussion, see Radach \& McConkie, 1998; Rayner \& Morris, 1992; Rayner, Sereno, \& Raney, 1996). Note that it is not necessary to assume that readers actually target a specific location in the parafoveal word. PLP could be indirectly determined as a function of what readers know they can usually process in the parafovea. The question as to whether parafoveal processing can influence landing position on line in reading (e.g., as a function of the actual position of lexical or sublexical information in the parafoveal word) is much more controversial. Underwood, Clews, and Everatt (1990) and Hyönä and Pollatsek $(1998,2000)$ have presented data suggesting that this might be the case. However, Underwood et al.'s finding was challenged by Rayner and Morris, who failed to replicate it when using nearly identical stimuli.

What about the relationship between landing position and recognition performance? Clearly, if the PLP effect is motivated by high-level processing constraints, some sort of penalty should be observed whenever the eyes happen to land elsewhere. The data concerning this question are not clear-cut either. A first set of apparently relevant exper- 
imental results were obtained with isolated word presentation. Word recognition speed has been shown to be dependent on what letter in the word is fixated. There is an optimal viewing position (OVP), ${ }^{1}$ located slightly left of center, at which word identification is easiest (O'Regan \& Jacobs, 1992; O'Regan et al., 1984; Vitu et al., 1990). This position is optimal for word recognition because it lowers the probability of refixation and thus shortens recognition time, with a delay of $20 \mathrm{msec}$ for each letter away from this position. Note, however, that the OVP is closer to the word middle than the PLP is. Moreover, the relationship between first fixation position and gaze duration is significantly attenuated or eliminated in normal reading (Rayner et al., 1996; Vitu et al., 1990).

Despite this discrepancy between the isolated word and the normal reading data, it may be important to note that the OVP, like the PLP, is dependent on reading habits (both are right of center for languages read from right to left; Deutsch \& Rayner, 1999), which can be seen as an argument that relates the two phenomena. Following this line of reasoning, the OVP effect may be partly explained by the left-right spatial structure of such languages as English and French. Similarly, the PLP effect may not be a strictly oculomotor phenomenon but, rather, the result of several combined factors, some of which are also at work in isolated word presentation. The main purpose of the present study was to further investigate this notion. However, it can be argued that the OVP and the PLP are not related to the same mechanism and that landing positions in reading are mainly the result of the properties of the oculomotor system. In most studies in which the OVP effect has been investigated, the position of the first fixation was imposed on participants (and systematically varied), so that no entering saccade was actually executed and no landing position was actually recorded. The OVP was inferred by means of an indirect measure-namely, the probability of making a refixation. This measure seems of little relevance to the PLP, since relatively few refixations are usually recorded in natural reading (McConkie, Kerr, Reddix, Zola, \& Jacobs, 1989; Rayner et al., 1996). In response to this objection, the procedure we used differed in one critical respect from the one used in the OVP literature. The initial fixation position in the stimulus was not imposed. Instead of being presented foveally, the stimulus appeared in the parafovea, either left or right of a central fixation point, and the participants were led to move their eyes so as to be in a more convenient position for performing the task (see the Method section of each experiment). Although this technique made it possible to directly record landing positions in isolated words and to partly control for the possible influence of oculomotor constraints, we are aware that it can be considered only as a remote analogue of natural reading (see Rayner, Fischer, $\&$ Pollatsek, 1998, for an attempt to address the PLP issue in a more natural situation). Remember, however, that one of the hypotheses under consideration in this study was precisely that some of the factors responsible for the PLP effect are also at work in isolated word processing. If PLP off-centeredness is found in the present study, whereas none of the factors specific to continuous reading are likely to enter into play, this would strengthen this hypothesis.

Following Radach, Krummenacher, Heller, and Hofmeister (1995), two alternative accounts of the PLP effect will be considered. The first states that the PLP effect is partly explained by a tendency for readers to undershoot when they are targeting the center of a word (low-level hypothesis). This hypothesis predicts a symmetrical distribution of the target landing positions on either side of the fixation point. More precisely, the eyes should land to the left of the middle when the target is presented on the right and to the right of the middle when it is presented on the left. ${ }^{2}$ According to the second hypothesis, a strategy implemented after a certain time lapse takes the eyes to the location that enables maximum information intake, irrespective of presentation side. In this case, one can expect the left/right pattern of landing positions to be asymmetrical, with leftward off-centeredness for both left and right presentations (processing adaptation hypothesis).

\section{EXPERIMENT 1}

The purpose of this experiment was to investigate the PLP effect for words and nonwords presented in isolation. Participants were asked to perform a lexical decision task on stimuli presented either left or right of an initial fixation point. This presentation mode, in conjunction with a task that can be assumed to involve a detailed visual analysis of the stimulus, was expected to favor the triggering of eye movements in the direction of the stimulus.

\section{Method}

Participants. Eighteen students from the University of Provence volunteered to participate. They were native speakers of French and had normal vision.

Apparatus. The participants saw linguistic stimuli displayed on the left or right side of a monitor interfaced with a PC computer placed about $60 \mathrm{~cm}$ in front of them, where 3.25 character spaces subtended $1^{\circ}$ of visual angle. Stimulus presentation was controlled by means of a button connected to the computer. The stimuli were displayed on the screen in lowercase white letters on a black background. Eye movements were collected by an infrared eyetracker (the Dr. Bouis Pupil-Centre Computation Oculometer) interfaced to a 12-bit A/D board. The position of the right eye was sampled every $5 \mathrm{msec}$. The participants' heads were held stationary by a headrest and a dental compound device upon which the upper jaw rested. The dental device was fastened to a metal plate that the participants could adjust in any of the three spatial planes. Prior to each block of four trials, the participants underwent a calibration procedure. They were asked to look one at a time at five points located in a line in the area to be occupied by the stimulus material. The overall accuracy of the system was approximately \pm 0.5 characters.

Materials. Ninety-six 9-character linguistic stimuli were used. In order to test for the influence of lexical access processes, the linguistic stimuli consisted of 48 words (mainly nouns and adjectives) and 48 nonwords. Most of the words were monomorphemic, with a proportion of polymorphemic adjectives. Fifty percent of the words were relatively infrequent (mean linguistic frequency of less than 27 occurrences per million, Trésor de la langue française, 1971), and $50 \%$ were frequent (mean frequency of greater than 133 occurrences 
per million). The orthographic regularity of the trigrams in the nonwords was also controlled. Half the nonwords were made up of relatively irregular trigrams, and the other half were made up of regular trigrams. A trigram was said to be irregular when few same-length words had that trigram in the same position (the mean sum of the trigram frequencies was $0.05 / 1,000)$ and to be regular when many same-length words had that trigram in the same position (mean sum, 7/1,000).

Task and Procedure. The participants were tested individually. They were asked to perform a lexical decision task on a series of letter strings that they caused to appear by pressing a button. At the beginning of each trial, they first had to fixate a colon, displayed in the middle of the screen, at the same time as they pressed a button that made the colon disappear and be replaced by a letter string. The letter string was displayed either on the right or on the left of the fixation point, in such a way that the nearest character in the stimulus was located two characters away from the fixation point. By pressing one of two keys, the participants had to decide as quickly and accurately as possible whether or not the stimulus was a French word (right button for yes, left button for no). An eight-item practice session was held in advance, followed by a single experimental list composed of words and nonwords (see Figure 1).

\section{Results and Discussion}

For nearly all the trials, the participants moved their eyes toward the stimulus, either left or right, before performing the lexical decision task. The question of interest for the present study is whether the size of these initial saccades was affected by the nature of the stimulus and the side of presentation. The results for words and nonwords as a function of type of stimulus (word vs. nonword) and presentation side (left vs. right) are summarized in Table 1. Mean saccade size, saccade latency, landing position, and lexical decision time are presented in columns 1,2,3, and 4 , respectively. As far as saccade size is concerned, the median of the overall distribution per condition is also given. Saccade size was measured relative to the position recorded while the participant was looking at the fixation point. Saccade direction was coded as a negative number for left presentations and as a positive number for right presentations. Landing position was measured relative to stimulus beginning (left edge). Saccade latency and lexical decision time were measured from the moment when the button was pressed (and the stimulus delivered). For the analysis, $5.33 \%$ of the trials were discarded because of a lack of eye movement, an initial saccade triggered in the wrong direction, or a change in the recorded position of the eye while the participant was looking at the fixation point (since a head movement was suspected in this case). Analyses of variance (ANOVAs) were conducted using a 2 (presentation side) $\times 2$ (type of stimulus) factorial design. Lexical frequency (high frequency vs. medium frequency) and trigram regularity were ignored, because of the outcome of prior analyses, conducted separately for words and nonwords, that revealed a lack of influence of these factors on saccade size [ $F<1$ for word frequency; $F(1,17)=2.73$, n.s., for trigram regularity; $F<1$ for the interaction of each of these factors with presentation side].

Saccade size. Saccade amplitude, rather than landing position, was chosen as the main dependent variable of this study, in order to allow small saccade size changes that might not have been picked up in the letter position metric to be observed (for discussions, see Inhoff, 1989, and Radach et al., 1995). However, since saccade and eye position data can be assumed to be dependent on the same processes, any variable that affects saccade size should also affect eye positioning. There was a main effect of pre-
Participants' Action

Example 1
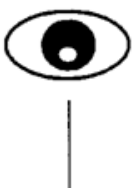

1st Button Pressing

EXPERIMENT 1:

2nd Button Pressing

Word/Non-word Answer

EXPERIMENTS 2-6:

2nd Button Pressing When

Subject Thought He/She Was in the Middle of the Stimulus

\section{Screen}

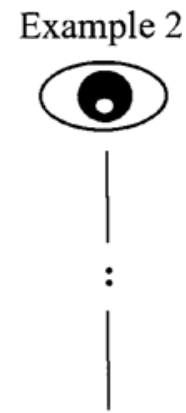

tombola

niplate
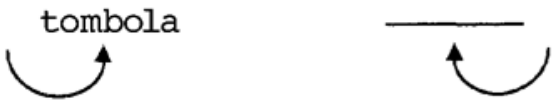

Figure 1. Diagram of the experimental task (Experiments 1-6). 
Table 1

Size and Direction of Initial Saccade (in Characters), Latency (in Milliseconds), Initial Landing Position (in Characters), and Lexical Decision Time (in Milliseconds) as a Function of Stimulus Type, Orthographic Familiarity, and Presentation Side (Experiment 1)

\begin{tabular}{|c|c|c|c|c|c|c|c|c|c|c|}
\hline \multirow[b]{3}{*}{ Stimulus } & \multicolumn{4}{|c|}{ Initial Saccade Size } & & & \multirow{2}{*}{\multicolumn{2}{|c|}{$\begin{array}{l}\text { Initial Landing } \\
\text { Position } \\
\end{array}$}} & \multirow{2}{*}{\multicolumn{2}{|c|}{$\begin{array}{c}\text { Lexical } \\
\text { DecisionTime } \\
\end{array}$}} \\
\hline & \multicolumn{2}{|c|}{ Left } & \multicolumn{2}{|c|}{ Right } & \multicolumn{2}{|c|}{ Latency } & & & & \\
\hline & $M$ & Median & $M$ & Median & $\overline{\text { Left }}$ & $\overline{\text { Right }}$ & Left & $\overline{\text { Right }}$ & Left & Right \\
\hline \multicolumn{11}{|l|}{ Words } \\
\hline High frequency & -6.9 & -7.0 & +5.0 & +5.0 & 161 & 171 & 4.1 & 4.0 & 772 & 764 \\
\hline Low frequency & -6.9 & -7.0 & +4.9 & +5.0 & 160 & 174 & 4.1 & 3.9 & 763 & 761 \\
\hline \multicolumn{11}{|l|}{ Nonwords } \\
\hline Regular & -6.9 & -7.0 & +4.9 & +5.0 & 162 & 167 & 4.1 & 3.9 & 784 & 781 \\
\hline Irregular & -6.8 & -7.0 & +4.9 & +5.0 & 161 & 169 & 4.2 & 3.9 & 786 & 780 \\
\hline
\end{tabular}

Note-Initial saccade size and direction were measured with respect to the central fixation point. The initial landing position was measured with respect to the beginning of the word. The mean standard deviations were similar across conditions (e.g., $0.68,0.62,0.67$, and 0.59 for initial saccade size). The mean standard deviations were about 18.8 for latency and 95.2 for lexical decision time.

sentation side [left vs. right; $F(1,17)=33.7, p<.001]$. Saccades were longer for left presentation than for right presentation, regardless of the type of stimulus (words or nonwords). The difference between words and nonwords was not significant $(F<1)$. When translated in terms of landing positions, this effect corresponded to the fact (visible in Table 1) that the mean landing position was located left of center for both left and right presentations. This finding is consistent with the existence of a PLP for linguistic stimuli presented in isolation and seems to support Radach et al.'s processing adaptation hypothesis. The participants apparently tended to reach a position located halfway between the middle and the left edge of the stimulus, whether saccading to the right or the left.

Saccade latency. The analysis of saccade latencies should shed some light on the mechanisms responsible for this effect. According to the processing adaptation hypothesis, the observed left/right asymmetry in saccade size is the result of some kind of high-level saccade positioning strategy (e.g., so that the eyes land where lexical information was expected to be found). Since instantiating such a high-level strategy presumably takes some time, relatively long saccade latencies could be predicted. The mean values presented in Table 1 do not support this prediction (overall mean $=166 \mathrm{msec}$ ). Moreover, it should be noted that no significant effects were revealed by the ANOVA on saccade latencies $[F(1,17)=1.70$, n.s., for the main effect of presentation side; all other $F_{\mathrm{S}}<1$ ]. Such a lack of a difference does not say anything about the strategies used by individual participants, however. It might be the case that the observed asymmetry (associated with long latencies) was present only for a subset of the participants. Radach et al. (1995) found only 1 participant (out of 4) whose results could be explained by a high-level strategy. In order to examine this possibility, each participant was assigned an asymmetry index (the difference between the mean sizes of his or her initial left and right saccades) and a rapidity index (his or her mean saccade latency, either left or right directed). The correlation between these two measures was negative $[r(16)=-.37, p=.12]$, and al- though it was nonsignificant, there was a trend that was repeated in subsequent experiments (some of which reached significance), which suggests that those participants who exhibited a large left/right saccade asymmetry were the ones whose mean saccade latencies were the shortest. The same tendency was found when separate analyses were made for words and nonwords $[r(16)=$ $-.38, p=.11$, and $r(16)=.39, p=.10$, respectively]. Clearly, this result does not support the high-level strategy interpretation (a positive correlation, with strong asymmetries associated with long latencies, was expected).

Lexical decision time. The analysis of lexical decision times revealed a significant main effect of stimulus type [words vs. nonwords; $F(1,17)=17.65, p<.001]$ and no stimulus type $\times$ presentation side interaction $(F<1)$. In order to further investigate the relation between landing position and OVP (defined here as the optimal viewing position for performing the lexical decision task), lexical decision times were examined as a function of initial landing position. This was possible only for Positions 3,4 , and 5 (relative to the stimulus left edge), which attracted more than $60 \%$ of the left saccades and more than $75 \%$ of the right saccades. There were not enough saccades that fell on other positions to allow for comparisons. For the three positions that had enough data, contrasting results were found for left and right presentations. For left presentations, a significant difference was found between the three positions [mean lexical decision time $=809,750$, and 729 msec for Positions 3, 4, and 5, respectively; $F(2,34)=$ $124.6, p<.001]$. Pairwise comparisons revealed a significant difference between all three positions $[F(1,34)=$ $46.19, p<.001$, for Position 3 vs. Position $4 ; F(1,34)=$ $83.44, p<.01$, for Position 3 vs. Position 5; and $F(1,34)$ $=4.68, p<.05$, for Position 4 vs. Position 5]. PLP and OVP clearly did not coincide in this experiment. Although the mean preferred landing position was approximately Position 4 for left presentations, Position 5 (which was the center of the word) was, in fact, the one that allowed for the quickest lexical decisions. This pattern of results sug- 
gests that landing positions are not determined mainly by lexical constraints, at least as far as left presentations are concerned. By contrast, few differences were observed for the three landing positions on the right side (mean lexical time $=730,731$, and $719 \mathrm{msec}$ for Positions 3, 4, and 5 respectively; $F<1$ ). None of the three pairwise comparisons was significant (all $F_{\mathrm{S}}<1$ ).

The most important result of this first experiment was the asymmetry found in saccade size for left and right presentations. Despite the results for saccade latency and lexical decision time, the possibility remains that part of the observed effect was related to the importance of word beginnings in reading, since it is a statistical fact that, in languages such as French and English, word beginnings are more lexically constraining than are word endings. In order to further examine this idea, Experiment 2 compared landing positions for isolated linguistic and nonlinguistic stimuli. Another possible interpretation for the observed asymmetry in terms of poor assessment of the middle of words can be proposed. More specifically, the participants may have aimed for the middle of the stimulus but landed to the left, owing to an erroneous perceptual evaluation of the stimulus midpoint. In favor of this view, a similar asymmetry has been observed in perception experiments in which linguistic and nonlinguistic stimuli were presented foveally. In an experiment using the classical bisection task (French participants were asked to indicate the middle of a solid line-i.e., the point on either side of which they saw two equal-length parts), Chokron and Imbert (1993) reported a tendency to place the midpoint to the left of the true midpoint. A similar effect was found by Fischer (1996) for English words. In order to investigate the possible influence of such a perceptual bias on landing position, a variant of the bisection task was used in Experiment 2: The participants were asked to look at the middle of stimuli and to validate the position by pressing a button.

\section{EXPERIMENT 2}

The purpose of this second experiment was twofold: (1) to compare landing positions for linguistic and nonlinguistic stimuli and (2) to compare landing positions and subjective midpoints. As in Experiment 1, the stimuli were presented in isolation, either to the left or to the right of an initial fixation point. The participants were asked to move their eyes as quickly as possible to a position they thought to be the middle of the stimulus and to validate this position by pressing a button.

\section{Method}

Participants. Sixteen students at the University of Provence volunteered to participate. All were native speakers of French with normal vision, and none had participated in Experiment 1.

Materials. The participants were presented with a series of 96 words and nonwords and a series of 96 solid lines. One series was composed solely of linguistic stimuli, and the other was composed solely of nonlinguistic stimuli. The linguistic stimuli included 24 low-frequency words (mean value, 10 occurrences per million), 24 high-frequency words ( 300 per million), 24 nonwords composed of relatively irregular trigrams (mean positional probability, $0.01 /$ $1,000)$, and 24 nonwords composed of regular trigrams $(8 / 1,000)$. In each condition, half the stimuli were 7 characters long, and the other half were 11 characters long. Similarly, half the solid lines were short (i.e., the length of a 7-character word), and the other half were long (the length of an 11-character word). As in Experiment 1, the stimuli were displayed either on the left or on the right of a fixation point in the middle of the screen, in such a way that either the left or the right end of the stimulus was located two character positions away from the fixation point.

Apparatus and Procedure. The apparatus was the same as that in Experiment 1. After the participant had fixated the colon displayed in the middle of the screen and had recorded that position by pressing a button, the colon was replaced by a stimulus (either a letter string or a solid line) displayed on the right or the left of the fixation point. The participants had to look at the location they felt was the middle of the stimulus and to record that position by pressing the button, as above. The recorded subjective middle was the location that was fixated when they pushed the button. They were instructed

Table 2

Size and Direction of Initial Saccade (in Characters), Latency (in Milliseconds), Initial Landing Position (in Characters), and Location of the Subjective Midpoint (in Characters) as a Function of Stimulus Type, Length, and Presentation Side (Experiment 2)

\begin{tabular}{|c|c|c|c|c|c|c|c|c|c|c|}
\hline \multirow[b]{3}{*}{ Stimulus } & \multicolumn{4}{|c|}{ Initial Saccade Size } & & & \multirow{2}{*}{\multicolumn{2}{|c|}{$\begin{array}{l}\text { Initial Landing } \\
\text { Position }\end{array}$}} & \multirow{2}{*}{\multicolumn{2}{|c|}{$\begin{array}{l}\text { Subjective } \\
\text { Midpoint }\end{array}$}} \\
\hline & \multicolumn{2}{|c|}{ Left } & \multicolumn{2}{|c|}{ Right } & \multicolumn{2}{|c|}{ Latency } & & & & \\
\hline & $M$ & Median & $M$ & Median & $\overline{\text { Left }}$ & Right & Left & Right & Left & Right \\
\hline \multicolumn{11}{|l|}{ Words } \\
\hline 7 characters & -5.1 & -5.0 & +4.3 & +4.0 & 159 & 165 & 3.9 & 3.3 & 4.7 & 3.3 \\
\hline 11 characters & -7.2 & -7.0 & +5.1 & +5.0 & 161 & 164 & 5.8 & 4.1 & 6.6 & 5.2 \\
\hline \multicolumn{11}{|l|}{ Nonwords } \\
\hline 7 characters & -5.2 & -5.0 & +4.4 & +4.0 & 160 & 165 & 3.8 & 3.4 & 4.7 & 3.3 \\
\hline 11 characters & -7.1 & -7.0 & +5.1 & +5.0 & 161 & 164 & 5.9 & 4.1 & 6.8 & 5.2 \\
\hline \multicolumn{11}{|l|}{ Solid lines } \\
\hline 7 characters long & -4.9 & -5.0 & +4.9 & +5.0 & 205 & 209 & 4.1 & 3.9 & 4.6 & 3.3 \\
\hline 11 characters long & -6.5 & -6.0 & +5.8 & +6.0 & 202 & 214 & 6.5 & 4.8 & 6.6 & 5.2 \\
\hline
\end{tabular}

Note-Initial saccade size and direction were measured with respect to the central fixation point. The initial landing position and the subjective midpoint were measured with respect to the beginning of the word. The mean standard deviations were similar across conditions (e.g., $0.56,0.50,0.55,0.55,0.54$, and 0.53 for initial saccade size). The mean standard deviations were about 21.9 for latency and 0.6 for subjective midpoint. 
to respond as quickly and as accurately as possible. The experiment comprised two experimental sessions, one for linguistic stimuli and one for nonlinguistic stimuli. The order of the two sessions was counterbalanced across participants. Half of them saw the linguistic stimuli first, and the other half saw the nonlinguistic stimuli first. An eight-item training phase was held at the beginning of each session.

\section{Results and Discussion}

For a majority of the trials, two successive saccades were recorded. In other words, the participantsfirst moved their eyes to an initial position in the stimulus and then made a corrective saccade in order to reach what they thought was the middle of the stimulus. Both the size of the initial saccade and the location selected as the middle (subjective midpoint) were measured. The mean values per condition are presented in Table 2, along with mean landing position and mean initial saccade latency. As in Experiment 1 , the initial saccade was measured with respect to the central fixation point, and landing position was measured with respect to stimulus beginning (i.e., left side of stimulus). The location of the subjective midpoint was measured relative to the stimulus beginning. All three measures were expressed in number of characters. Saccade latency was expressed in milliseconds and was measured relative to the moment when the button was first pressed. As in Experiment 1, only those trials eliciting a saccade in the right direction (and void of any head movement) were included in the analysis $(4.91 \%$ of the trials were discarded).

Saccade size. As a first step, separate ANOVAs were carried out for words and nonwords. No lexical frequency effect for words and no orthographic regularity effect for nonword trigrams were found $(F \mathrm{~s}<1)$. A combined analysis was then conducted, with presentation side (left vs. right) and type of stimulus (words vs. nonwords vs. solid lines) as the main factors. Stimulus length was also included in the analysis but will be mentioned in the discussion of the results only when it interacted with the other two factors. There was a significant interaction between the type of stimulus and the presentation side $[F(2,28)=19.67, p<.001]$. Pairwise comparisons revealed that the interaction could be explained entirely by the fact that there was a difference between linguistic stimuli (words and nonwords) and nonlinguistic stimuli [solid lines; $F(1,28)=37.92, p<.001$ ] and no difference between words and nonwords $(F<1)$. This pattern of results suggests that different strategies were used for linguistic and nonlinguistic stimuli. As is indicated in Table 2 (column 3), the mean landing position for both words and nonwords was close to the stimulus middle for left presentations and was located halfway between the beginning and the middle of the stimulus when the saccade was rightward (i.e., when the participants were moving their eyes to the right, as they typically do in reading). By contrast, left and right landing positions were approximately symmetric for nonlinguistic stimuli.

The ANOVA also revealed an interaction between presentation side and stimulus length $[F(1,14)=51.14, p<$
.001], which corresponded to the fact, visible in Table 2, that (1) the observed asymmetry was more marked for long linguistic stimuli than for short ones and (2) a hint of asymmetry was possibly present for long nonlinguistic stimuli as well. Note, however, that no left/right difference was observed in the latter condition as far as medians are concerned.

Initial saccade latency. The ANOVA yielded a main effect of type of stimulus $[F(2,28)=148.70, p<.001]$. Pairwise comparisons revealed that the effect could be explained entirely by the difference between linguistic stimuli (words and nonwords) and nonlinguistic stimuli [solid lines; $162 \mathrm{msec}$ for linguistic stimuli vs. $208 \mathrm{msec}$ for nonlinguistic stimuli, $F(1,28)=297.17, p<.001]$ and that the difference between words and nonwords was nonsignificant $(F<1)$. It is interesting to note that Rayner and Fischer (1996) obtained a similar pattern, with shorter fixation durations in the normal-text-reading condition than in the $z$-text-reading condition ( 279 vs. $317 \mathrm{msec}$ ). These results are also consistent with those obtained by Vitu, O'Regan, Inhoff, and Topolski (1995) and Fischer (1999). The fact that longer initial saccade latencies were found for stimuli that exhibited a symmetrical landing position pattern for left and right presentations-namely, nonlinguistic stimuli-is at odds with Radach's (Radach et al., 1995) high-level strategy hypothesis. In this view, symmetrical patterns are the result of low-level factors and should be associated with short latencies, whereas asymmetrical patterns are produced by knowledge-level factors and should be associated with long latencies. In order to further investigate this question, an asymmetry index and a rapidity index were calculated for each subject and each type of stimulus (see Experiment 1 for details). A small negative correlation was found again for words $[r(14)=-.42, p=.10]$, which is in line with the result pattern for the above comparison of stimulus types. No effect was found for nonwords or solid lines $[r(14)=-.17$, $p=.53$, and $r(14)=-.12, p=.66$, respectively].

Subjective midpoint. As Table 2 shows, the participants tended to mislocate the midpoint, no matter what type of stimulus was presented (words, nonwords, or solid lines). The position that the participants considered to be the middle of the stimulus was located left of center for right presentations and right of center for left presentations. In other words, the subjective midpoint was farther away from the left edge of the stimulus for left presentation, as compared with right presentation $[F(1,14)=66.48$, $p<.001]$. No interaction involving presentation side reached the significance level (all $F \mathrm{~s}<1$ or close to 1 ).

The results for right presentation are consistent with previous findings of a leftward bias in classical bisection experiments (Chokron \& Imbert, 1993). Unlike Fischer (1996), we found no differences between the biases observed on words, nonwords, and lines $(F<1)$. The important fact, visible in Table 2, is that landing positions and subjective midpoints did not exactly coincide in Experiment 2 . This suggests that the explanation in terms of 
perceptual bias (i.e., poor evaluation of the stimulus middle) can probably be rejected. Apparently, the participants knew that the position they landed on was not the middle, since they usually made a corrective saccade before pressing the button. ${ }^{3}$

As in Experiment 1, a left/right initial saccade asymmetry was nevertheless found for linguistic stimuli. By contrast, symmetrical landing positions were found for solid lines. It is true that the asymmetry obtained for linguistic stimuli was less pronounced than in Experiment 1. This can probably be explained by task differences. Remember that the participants were explicitly required to aim for the middle in Experiment 2. In such a situation, similar initial landing positions (i.e., close to the middle of the stimulus) could have been expected for all conditions, whatever the type of stimulus and the presentation side. A difference was nevertheless observed between linguistic and nonlinguistic stimuli. There thus seems to be something special about linguistic stimuli that is not present in solid lines and that can probably account for the observed left/right asymmetry. Part of the answer might lie in the fact that linguistic stimuli, unlike solid lines, are composed of discrete elements (namely, characters). This, rather than the distribution of linguistic information, could be responsible for the triggering of a specific left-to-right visual scanning strategy. This possibility was examined in Experiment 3.

\section{EXPERIMENT 3}

The results of Experiment 2 brought out a number of landing position differences between linguistic and nonlinguistic stimuli. However, the question remained as to the cause of these differences. Are they linked to the linguistic nature of words and nonwords (i.e., the presence of letters and legal clusters of letters) or simply to the discreteness of this type of stimulus (i.e., the fact that letters are discrete elements) as opposed to the continuousness of solid lines? In order to address this question, the nonwords from Experiment 2 were replaced by strings of hashes (\#\#\#\#\#\#) in Experiment 3. Experiment 3 was similar to Experiment 2 in other respects, with again three types of stimuli-namely, words, strings of hashes, and solid lines. Strings of hashes were useful here because they are both nonlinguistic (like solid lines) and composed of discrete elements (like words). By comparing the three conditions, it should be possible to determine which factor was responsible for the asymmetry found in Experiment 2.

\section{Method}

Participants. Sixteen freshmen students at the University of Provence participated in the experiment. All were native speakers of French and had normal vision without corrective lenses. None of them had participated in Experiment 1 or 2.

Materials. Two lists were used. The first was composed of 96 words, $50 \%$ of which were relatively infrequent (fewer than 29 occurrences per million) and $50 \%$ of which were frequent (more than 350 occurrences per million). The second contained 48 strings of hashes and 48 solid lines. As in Experiment 2, the stimuli were 7 and 11 characters long.

Procedure. The task and procedure were identical to those used in Experiment 2. The experiment comprised two successive sessions corresponding to the two experimental lists. During one session, the participants were presented only with words, whereas during the other, they were presented with strings of hashes and solid lines in random order. An eight-item training phase was run at the beginning of each session. The presentation order of the two sessions (experimental lists) was counterbalanced across participants.

\section{Results and Discussion}

As in Experiment 2, both the size of the initial saccade and the location selected as the middle of the stimulus (subjective midpoint) were measured. The mean values per condition are presented in Table 3, along with mean landing position and mean initial saccade latency $(4.58 \%$ of the trials were discarded).

Saccade size. A separate ANOVA conducted on the linguistic stimuli did not reveal any word frequency effect

Table 3

Size and Direction of Initial Saccade (in Characters), Latency (in Milliseconds), Initial Landing Position (in Characters), and Location of the Subjective Midpoint (in Characters) as a Function of Stimulus Type, Length, and Presentation Side (Experiment 3)

\begin{tabular}{|c|c|c|c|c|c|c|c|c|c|c|}
\hline \multirow[b]{3}{*}{ Stimulus } & \multicolumn{4}{|c|}{ Initial Saccade Size } & \multirow{2}{*}{\multicolumn{2}{|c|}{ Latency }} & \multirow{2}{*}{\multicolumn{2}{|c|}{$\begin{array}{l}\text { Initial Landing } \\
\text { Position } \\
\end{array}$}} & \multirow{2}{*}{\multicolumn{2}{|c|}{$\begin{array}{c}\text { Subjective } \\
\text { Midpoint }\end{array}$}} \\
\hline & \multicolumn{2}{|c|}{ Left } & \multicolumn{2}{|c|}{ Right } & & & & & & \\
\hline & $M$ & Median & $M$ & Median & Left & Right & Left & Right & $\overline{\text { Left }}$ & Right \\
\hline \multicolumn{11}{|l|}{ Words } \\
\hline 7 characters & -5.3 & -5.0 & +4.3 & +4.0 & 158 & 164 & 3.7 & 3.3 & 4.7 & 3.3 \\
\hline 11 characters & -7.2 & -7.0 & +5.3 & +5.0 & 162 & 163 & 5.8 & 4.3 & 6.6 & 5.3 \\
\hline \multicolumn{11}{|l|}{ Strings of hashes } \\
\hline 7 characters long & -5.1 & -5.0 & +4.3 & +4.0 & 182 & 189 & 3.9 & 3.3 & 4.6 & 3.4 \\
\hline 11 characters long & -7.1 & -7.0 & +5.2 & +5.0 & 182 & 188 & 5.9 & 4.2 & 6.6 & 5.4 \\
\hline \multicolumn{11}{|l|}{ Solid lines } \\
\hline 7 characters long & -4.5 & -5.0 & +4.5 & +5.0 & 188 & 195 & 4.5 & 3.5 & 4.6 & 3.5 \\
\hline 11 characters long & -5.8 & -6.0 & +5.6 & +6.0 & 190 & 197 & 7.2 & 4.6 & 6.6 & 5.5 \\
\hline
\end{tabular}

Note-Initial saccade size and direction were measured with respect to the central fixation point. The initial landing position and the subjective midpoint were measured with respect to the beginning of the word. The mean standard deviations were similar across conditions (e.g., $0.47,0.48,0.44,0.47,0.50$, and 0.51 for initial saccade size). The mean standard deviations were about 22.1 for latency and 0.49 for subjective midpoint. 
$(F<1)$. This factor was no longer taken into account in the full analysis that combined all three types of stimuli. The data in Table 3 indicate that initial-saccade size was similar for words and strings of hashes but was different for solid lines. A significant interaction was found between stimulus type and presentation side $[F(2,28)=31.9$, $p<.001]$. Pairwise comparisons were used to analyze this interaction in greater detail. They revealed that the difference between words and strings of hashes was nonsignificant $[F(1,28)=1.17$, n.s. $]$ and that the interaction between stimulus type and presentation side could be explained entirely by the strong opposition between discrete stimuli (words and strings of hashes) and continuous stimuli [solid lines; $F(1,28)=47.24, p<.001$ ], thus confirming the importance of stimulus discreteness versus continuousness in determining landing position.

Latency. As one can see in Table 3, the latencies of incoming saccades were similar to those observed in Experiment 2. There was a significant main effect of stimulus type $[F(2,28)=84.30, p<.001]$. This difference was explained mainly by the contrast between linguistic and nonlinguistic stimuli, with shorter latencies for linguistic stimuli than for nonlinguistic stimuli $[F(1,28)=110.89$, $p<.001]$. The opposition between discrete and continuous stimuli was marginal, however $[F(1,28)=4.04, p=$ $.06]$. This pattern of results seems to rule out the possibility of any high-level strategy for linguistic stimuli, a conclusion that was also supported by the analysis of individual strategies. As in the previous experiments, both asymmetry and rapidity indexes were calculated for each participant and each type of stimulus. The results were similar to those of Experiment 2-namely, a negative correlation for words $[r(14)=-.52, p=.05]$ and a lack of correlation for strings of hashes $[r(14)=-.09$, n.s. $]$ and solid lines $[r(14)=-.29$, n.s. $]$.

Subjective midpoint. As in Experiment 2, in the majority of cases, the location that the participants considered to be the middle of the stimulus was not exactly the same as the initial fixation position. This location was farther away from the stimulus left edge for left than for right presentation $[F(1,14)=83.44, p<.001]$. A rightward bias was observed for left presentation, whereas a leftward bias was observed for right presentation. This was true whether the stimulus was discrete or continuous $(F<1$ for the interaction between presentation side and type of stimulus). This lack of interaction is at odds with Fischer's (1996) conclusion that a leftward bias can be observed on pseudowords (as opposed to illegal strings of characters), because, in the pseudoword case, the participants were expecting to see a word. In the present case, linguistic and nonlinguistic stimuli were presented in separate sessions. The possibility that the participants were expecting a word instead of a nonlinguistic stimulus can thus be ruled out.

\section{EXPERIMENT 4}

The purpose of this experiment was to make sure that the asymmetry found for strings of hashes (as opposed to solid lines) was not caused by some visual property specific to this type of stimulus. In addition to being composed of discrete elements, strings of hashes differ from solid lines in size and brightness, and it could be argued that these factors, rather than discreteness, were responsible for the observed differences. In order to address this potential criticism, strings of hashes were replaced by dotted lines in Experiment 4. Moreover, since no difference was found between strings of hashes and words in Experiment 3, we decided to limit the materials to nonlinguistic stimuli in Experiment 4. The comparison was thus between dotted and solid lines, presented either left or right of a central fixation point.

\section{Method}

Participants. Sixteen students from the University of Provence volunteered to participate. All were native speakers of French and had normal vision. None of the participants was in any of the preceding experiments.

Materials. The materials consisted of 160 nonlinguistic stimuli, 7 and 11 characters long: 80 solid lines and 80 dotted lines. They were presented either 2 characters to the left or 2 characters to the right of the central fixation point.

Procedure. The task and procedure were similar to those used in Experiments 2 and 3. The experiment comprised two sessions. Each

Table 4

Size and Direction of Initial Saccade (in Characters), Latency (in Milliseconds), Initial Landing Position (in Characters), and Location of the Subjective Midpoint (in Characters) as a Function of Stimulus Type, Length, and Presentation Side (Experiment 4)

\begin{tabular}{|c|c|c|c|c|c|c|c|c|c|c|}
\hline \multirow[b]{3}{*}{ Stimulus } & \multicolumn{4}{|c|}{ Initial Saccade Size } & \multirow{2}{*}{\multicolumn{2}{|c|}{ Latency }} & \multirow{2}{*}{\multicolumn{2}{|c|}{$\begin{array}{l}\text { Initial Landing } \\
\text { Position } \\
\end{array}$}} & \multirow{2}{*}{\multicolumn{2}{|c|}{$\begin{array}{c}\text { Subjective } \\
\text { Midpoint }\end{array}$}} \\
\hline & \multicolumn{2}{|c|}{ Left } & \multicolumn{2}{|c|}{ Right } & & & & & & \\
\hline & $M$ & Median & $M$ & Median & Left & Right & Left & Right & Left & Right \\
\hline \multicolumn{11}{|l|}{ Dotted lines } \\
\hline 7 characters long & -4.6 & -5.0 & +3.9 & +4.0 & 198 & 195 & 4.4 & 2.9 & 4.5 & 3.4 \\
\hline 11 characters long & -6.6 & -7.0 & +5.2 & +5.0 & 196 & 196 & 6.4 & 4.2 & 6.6 & 5.4 \\
\hline \multicolumn{11}{|l|}{ Solid lines } \\
\hline 7 characters long & -4.2 & -4.0 & +4.3 & +4.0 & 195 & 197 & 4.8 & 3.3 & 4.5 & 3.4 \\
\hline 11 characters long & -6.2 & -6.0 & +5.8 & +6.0 & 187 & 196 & 6.8 & 4.8 & 6.5 & 5.5 \\
\hline
\end{tabular}

Note-Initial saccade size and direction were measured with respect to the central fixation point. The initial landing position and the subjective midpoint were measured with respect to the beginning of the word. The mean standard deviations were similar across conditions (e.g., 0.53, 0.54, 0.53, and 0.59 for initial saccade size). The mean standard deviations were about 24.9 for latency and 0.55 for subjective midpoint. 
session began with an eight-trial training phase. In one session, the stimuli were solid lines, and in the other, they were dotted lines. The order of the two sessions was counterbalanced across participants. Half of the participants began with the dotted lines, and the other half began with the solid lines.

\section{Results and Discussion}

The mean saccade size, saccade latency, landing position, and subjective midpoint per condition are given in Table $4(5.57 \%$ of the trials were discarded from the analysis). The results for saccade latency and subjective midpoint were consistent with what was found for nonlinguistic stimuli in the previous experiment [no significant effect for saccade latency, all $F \mathrm{~s}<1$ or close to 1 ; main effect of presentation side for the subjective midpoint analysis, $F(1,14)=11.07, p<.01]$. As for saccade size, the results nearly replicated those of Experiment 3 for nonlinguistic stimuli. As Table 4 shows, the mean left and right landing positions were asymmetrical for dotted lines and were nearly symmetrical for solid lines. In the latter case, the effect was clear for short stimuli only. An ANOVA conducted on incoming saccade size revealed a significant interaction between type of stimulus (dotted vs. solid lines) and presentation side $[F(1,14)=9.30, p<$ $.001]$ and a nearly significant three-way interaction between type of stimulus, presentation side, and stimulus length $[F(1,14)=3.82, p<.07]$.

The fact that a similar asymmetry was found for words (Experiments 1, 2, and 3), nonwords (Experiments 1 and 2), strings of hashes (Experiment 3), and dotted lines points out the importance of stimulus discreteness in explaining the phenomenon and allows us to rule out the possibility of a bias caused by some unknown property specific to a given type of stimulus. Considered as a whole, these experiments suggest that the participants used different inspection strategies, depending on whether the stimulus was discrete or continuous, irrespective of the type of task or the type of discrete stimulus. This result raises the intriguing question of knowing why the participants should have tried to land closer to the beginning (i.e., the left edge) when targeting nonlinguistic discrete stimuli. In the following experiment, we examined the possibility that this could be linked to reading habits.

\section{EXPERIMENT 5}

The purpose of this experiment was to determine whether the asymmetry found in left versus right landing positions for discrete stimuli can be explained in terms of reading habits. The participants were Arabic/French bilinguals who first read a text either in Arabic (right to left) or in French (left to right) and were then asked to perform a bisection task on a series of discrete and continuous stimuli presented to the left or right of an initial fixation point. Again, the measure of interest was the size of the incoming saccade as a function of type of stimulus and presentation side. The possible influence of reading habits was examined by comparing landing patterns (as indicated by saccade size) according to whether the participants read in Arabic or French prior to the experiment.

\section{Method}

Participants. Ten Arabic/French bilingual students from the University of Provence participated in the experiment. All had normal vision without corrective lenses. They were native speakers of Arabic and had received a bilingual education in France, where they had

Table 5

Size and Direction of Initial Saccade (in Characters), Latency (in Milliseconds), Initial Landing Position (in Characters), and Location of the Subjective Midpoint (in Characters) as a Function of Reading Direction, Stimulus Type, Length, and Presentation Side (Experiment 5)

\begin{tabular}{|c|c|c|c|c|c|c|c|c|c|c|}
\hline \multirow[b]{3}{*}{ Stimulus } & \multicolumn{4}{|c|}{ Initial Saccade Size } & \multirow{2}{*}{\multicolumn{2}{|c|}{ Latency }} & \multirow{2}{*}{\multicolumn{2}{|c|}{$\begin{array}{l}\text { Initial Landing } \\
\text { Position }\end{array}$}} & \multirow{2}{*}{\multicolumn{2}{|c|}{$\begin{array}{l}\text { Subjective } \\
\text { Midpoint }\end{array}$}} \\
\hline & \multicolumn{2}{|c|}{ Left } & \multicolumn{2}{|c|}{ Right } & & & & & & \\
\hline & $M$ & Median & $M$ & Median & Left & Right & Left & Right & Left & Right \\
\hline \multicolumn{11}{|l|}{ Left-to-right reading } \\
\hline \multicolumn{11}{|l|}{ Strings of hashes } \\
\hline 7 characters long & -5.1 & -5.0 & +4.2 & +4.0 & 183 & 181 & 3.9 & 3.2 & 4.5 & 3.6 \\
\hline 11 characters long & -6.7 & -7.0 & +4.8 & +5.0 & 182 & 201 & 6.3 & 3.8 & 6.4 & 5.6 \\
\hline \multicolumn{11}{|l|}{ Solid lines } \\
\hline 7 characters long & -4.8 & -5.0 & +4.8 & +5.0 & 208 & 199 & 4.2 & 3.8 & 4.5 & 3.5 \\
\hline 11 characters long & -5.5 & -5.0 & +5.3 & +5.0 & 213 & 204 & 7.5 & 4.3 & 6.5 & 5.5 \\
\hline \multicolumn{11}{|l|}{ Right-to-left reading } \\
\hline \multicolumn{11}{|l|}{ Strings of hashes } \\
\hline 7 characters long & -4.5 & -4.0 & +4.7 & +5.0 & 192 & 195 & 4.5 & 3.7 & 4.2 & 4.0 \\
\hline 11 characters long & -6.0 & -6.0 & +5.1 & +5.0 & 201 & 187 & 7.0 & 4.1 & 6.2 & 6.1 \\
\hline \multicolumn{11}{|l|}{ Solid lines } \\
\hline 7 characters long & -4.4 & -4.0 & +4.4 & +4.0 & 213 & 205 & 4.6 & 3.4 & 4.1 & 4.1 \\
\hline 11 characters long & -5.6 & -6.0 & +5.5 & +5.0 & 227 & 213 & 7.4 & 4.5 & 6.1 & 6.1 \\
\hline
\end{tabular}

Note-Initial saccade size and direction were measured with respect to the central fixation point. The initial landing position and the subjective midpoint were measured with respect to the beginning of the word. The mean standard deviations were similar across conditions (e.g., $0.63,0.45,0.60,0.52,0.63,0.49,0.59$, and 0.50 for initial saccade size). The mean standard deviations were about 23.0 for latency and 0.53 for subjective midpoint. 
been brought up. None of the participants was aware of the purpose of the experiment.

Materials. The materials consisted of 160 nonlinguistic stimuli that were 7 or 11 characters long: 80 solid lines and 80 strings of hashes.

Procedure. In order to induce a reading direction for the stimuli, the participants were first asked to read a text either in Arabic (rightto-left reading) or in French (left-to-right reading; training phase). Then they performed a bisection task, following the procedure used in the preceding experiments (except for the direction of the calibration phase, which was the same as the imposed reading direction in the training phase). ${ }^{4}$ Nonlinguistic stimuli (strings of hashes and solid lines) were displayed on the left or the right of the central fixation point. The participants were asked to look at the place they felt was the middle of the stimulus and to record that position by pressing a button. The experiment comprised two sessions. In one session, the participants were presented with solid lines, and in the other, with strings of hashes. The order of the sessions was counterbalanced across participants. Eight practice items were proposed at the beginning of each session.

\section{Results and Discussion}

No data were recorded during the training phase. The results summarized in Table 5 (saccade size and latency, landing position, and subjective midpoint) were obtained from the data recorded during the bisection task $(5.08 \%$ of the trials were discarded from the analysis).

Saccade size. The analysis of incoming saccade size yielded a three-way interaction between the reading direction imposed during the training session (left to right vs. right to left), the type of stimulus (discrete vs. continuous), and the presentation side [left vs. right; $F(1,8)=7.09, p<$ .05]. Separate analyses for French and Arabic training indicated that the stimulus type $\times$ presentation side interaction was significant in the former condition only $[F(1,8)=$ $47.34, p<.001$; with $F<1$ in the latter condition], suggesting that landing position patterns varied as a function of the reading direction imposed during training. As Table 5 shows, when the participants had just been trained in French (left-to-right reading), the landing position pattern was comparable to that of the monolingualFrench participants in the previous experiments (left/right asymmetry for discrete stimuli, but not for continuous stimuli). Inducing a right-to-left exploration direction during training eliminated the landing asymmetry for discrete stimuli (symmetrical landing pattern for both types of stimuli). One can hypothesize that the left/right asymmetry was not reversed in the Arabic training condition because the bilinguals who participated in this experiment were more accustomed to reading in French than in Arabic. Note, however, that landing positions relative to the entering edge of the stimulus (i.e., the left edge for right presentation and the right edge for left presentation) were similar in the leftto-right training/right presentation condition and in the right-to-left training/left presentation condition. This result is consistent with those obtained by Deutsch and Rayner (1999), who demonstrated that the PLP effect for readers of Hebrew, a right-to-left language, closely resembles the PLP found for readers of left-to-right European languages. That is, readers of Hebrew and English tend to land initially about halfway between the beginning and the center of the word. Of course, for Hebrew readers, this means that they initially fixate to the right of the word's center.

Latency and subjective midpoint. As Table 5 shows, the pattern of results for saccade latencies was comparable to what was observed in Experiment 3 (even though the latencies for the right-to-left training condition were slightly longer). No significant effects were revealed by the ANOVA, however (all $F \mathrm{~s}<2.5$ ). As for subjective midpoints, contrasting results were obtained as a function of training direction. As one can see in Table 5, when the participants were initially led to read from left to right, the results were consistent with those obtained in Experiments 2 and 3, with a tendency to move the subjective midpoint toward the entering edge of the stimulus (approximately a half-character right displacement for left presentations vs. a half-character left displacement for right presentations). This bias disappeared when the participants had been trained in the right-to-left direction (the subjective midpoint nearly coincided with the true midpoint in this case, regardless of the side of presentation). The main effect of training was significant $[F(1,8)=$ $33.09, p<.001]$. The interaction between training and presentation side was marginally significant $[F(1,8)=$ $3.58, p=.10]$, whereas the three-way interaction between training, presentation side, and type of stimulus was not significant $[F(1,8)=1.46$, n.s.]. This pattern of results differs from those obtained by Chokron and Imbert (1993) and Chokron and de Agostini (1995), who demonstrated the existence of a rightward bias in the estimation of the subjective midpoint by monolingual Arabic and Israeli readers tested using the classical bisection task. However, remember that the participants in the present experiment were bilingual students (with a possible bias in favor of left-to-right reading), whereas Chokron and colleagues' participants were monolinguals. This can probably explain why a lack of bias (instead of the expected reversed bias) was observed here for the right-to-left training condition.

\section{EXPERIMENT 6}

Before drawing any strong conclusions from Experiment 5 , we needed to see whether a similar pattern of results would be obtained if monolingual readers were asked to move their eyes from right to left for a while before performing a bisection task. The aim of Experiment 6 was to address this question.

\section{Method}

Participants. Ten French monolingual students from the University of Provence volunteered to participate. They were native speakers of French and had normal vision without corrective lenses. None of them had participated in the previous experiments.

Materials and Procedure. The materials and procedure for the bisection task were the same as those in the previous experiment. In order to induce right-to-left scanning, the participants were first asked to perform a pencil-and-paper task in which they had to search for gaps in series of digits or letters presented in right-to-left numerical order (or in right-to-left alphabetical order). For example, in the following alphabetical series, "r q p o n $1 \mathrm{kj}$ i h g f e," they had 
Table 6

Size and Direction of Initial Saccade (in Characters), Latency (in Milliseconds), Initial Landing Position (in Characters), and Location of the Subjective Midpoint (in Characters) as a Function of Stimulus Type, Length, and Presentation Side for Right-to-Left Reading (Experiment 6)

\begin{tabular}{|c|c|c|c|c|c|c|c|c|c|c|}
\hline \multirow[b]{3}{*}{ Stimulus } & \multicolumn{4}{|c|}{ Initial Saccade Size } & & & \multirow{2}{*}{\multicolumn{2}{|c|}{$\begin{array}{l}\text { Initial Landing } \\
\text { Position } \\
\end{array}$}} & \multirow{2}{*}{\multicolumn{2}{|c|}{$\begin{array}{c}\text { Subjective } \\
\text { Midpoint }\end{array}$}} \\
\hline & \multicolumn{2}{|c|}{ Left } & \multicolumn{2}{|c|}{ Right } & \multicolumn{2}{|c|}{ Latency } & & & & \\
\hline & $M$ & Median & $M$ & Median & Left & Right & Left & Right & Left & Right \\
\hline \multicolumn{11}{|l|}{ Strings of hashes } \\
\hline 7 characters long & -4.9 & -5.0 & +4.7 & +5.0 & 197 & 183 & 4.1 & 3.7 & 4.2 & 3.9 \\
\hline 11 characters long & -6.6 & -7.0 & +5.8 & +6.0 & 206 & 186 & 6.4 & 4.8 & 5.8 & 6.1 \\
\hline \multicolumn{11}{|l|}{ Solid lines } \\
\hline 7 characters long & -4.4 & -5.0 & +4.4 & +4.0 & 225 & 214 & 4.6 & 3.4 & 4.0 & 3.9 \\
\hline 11 characters long & -5.9 & -6.0 & +5.6 & +6.0 & 234 & 219 & 7.1 & 4.6 & 5.9 & 5.9 \\
\hline
\end{tabular}

Note-Initial saccade size and direction were measured with respect to the central fixation point. The initial landing position and the subjective midpoint were measured with respect to the beginning of the word. The mean standard deviations were similar across conditions (e.g., 0.73, 0.68, 0.69, and 0.65 for initial saccade size). The mean standard deviations were about 27.8 for latency and 0.62 for subjective midpoint.

to put a slash between the letters "l" and " $n$ " because the letter " $m$ " was missing. Then they performed a bisection task, following the procedure used in the preceding experiment.

\section{Results and Discussion}

No data were recorded during the training session. The results in Table 6 were obtained from the bisection task data (6.05\% of the trials were discarded from the analysis).

Saccade size. The analysis of saccade size did not reveal a significant interaction between stimulus type and presentation side $[F(1,9)=1.77$, n.s. $]$. As Table 6 shows, the landing position pattern was comparable to that of the bilingual Arabic/French participants in the Arabic-training condition of Experiment 5. The landing position pattern found for French monolinguals in the previous experiments (left/right asymmetry in the discrete-stimulus case) was apparently eliminated here, presumably because of the right-to-left exploration direction induced during training. An ANOVA combining Experiments 3 and 6 was carried out (for discrete stimuli only). A significant interaction between experiment and presentation side was obtained $[F(1,23)=7.29, p=.01]$, thus confirming that different left/right distributions were found, depending on scanning direction (i.e., asymmetrical distribution in Experiment 3 vs. nearly symmetrical distribution in Experiment 6). This suggests that the asymmetry found in the previous experiments was not due to reading habits per se, since the effect disappeared when the participants were asked to move their eyes in a direction opposite to their usual reading direction for a short while (Experiment 6). A similar conclusion could be drawn from Inhoff, Pollatsek, Posner, and Rayner's (1989) paper. In an experiment comparing eye movement behavior in left-to-right versus right-to-left reading, they found little difference between the left-to-right and the right-to-left conditions in terms of overall reading performance. Native English readers were apparently able to make use of parafoveal information from the left of fixation (without practice) in the right-toleft condition. The authors argued in favor of an atten- tional spotlight, with the direction of attention shifts tied closely to the direction of eye movements.

Latency and subjective midpoint. No significant effect was revealed by the analysis of saccade latency [all $F$ s $<1$ or close to 1, except for the main effect of stimulus type, $F(1,9)=2.96$, n.s.]. Note that the latencies were longer in this experiment than in the first four (209 vs. $188 \mathrm{msec}$ on average). This tendency is probably related to the unusual nature of the task (French monolinguals were trained to move their eyes from right to left prior to the experiment, as well as during the calibration procedure). The lack of asymmetry found for saccade size was replicated in the subjective midpoint analysis. As one can see in Table 6 , the tendency to mislocate the midpoint tended to disappear here with right-to-left training $(F<1$ for the main effect of presentation side, as well as for the interaction between presentation side and stimulus type). These results are consistent with those obtained in the previous experiment in the right-to-left training condition. Note, however, that as in the other experiments, the subjective midpoint did not coincide with the landing position.

\section{GENERAL DISCUSSION}

The aim of the experiments reported in this paper was to investigate the physical and cognitive sources of control over the positioning of saccadic eye movements while participants processed isolated words and nonlinguistic stimuli. The results of the first experiment suggested the existence of an asymmetry in the extent of short saccades launched toward isolated words and nonwords lying either to the right or to the left of an initial fixation point, with left-going saccades about two characters longer. This is consistent with the finding that participants arrive somewhere left of center for both presentation sides and suggests that some of the factors responsible for the PLP effect (i.e., the fact that readers usually land between the beginning and the middle of words) are also at work in single-word targeting. Whether these factors relate to low- 
level oculomotor constraints or high-level positioning strategies was examined in the experiments that followed. Experiment 2 contrasted words with continuous lines of the same length. The participants were instructed to attempt to fixate the exact center of the displayed stimulus (a task typically demanding a corrective saccade after landing). The results showed that the obtained asymmetry was restricted to linguistic stimuli: Saccade extent for continuous lines was roughly equivalent in both directions. Experiment 3 was an attempt to untangle physical and linguistic sources of influence over this asymmetry. In different conditions, participants directed saccades toward words, continuous lines, or strings of hashes (\#\#\#\#\#\#). The sharp-sequence stimuli, although clearly meaningless, were nonetheless composed of discrete characters. The results were clear-cut: The asymmetry was restricted to (and equivalent in size in) the character-based strings (words and hashes) and was absent for continuous lines. Experiment 4 further supported this finding by demonstrating a similar substantial asymmetry for dotted lines (------). The results of these first four experiments do not seem to fit with any of the hypotheses mentioned in the introduction. The low-level perceptual and/or oculomotor account and the high-level knowledge-dependentaccount are discussed below.

The results do not support the view that basic oculomotor and/or perceptual constraints associated with saccade programming and execution are the sole determinants of landing position in isolated stimuli. The left/right asymmetry found for some types of stimuli (i.e., words and nonwords in Experiments 1 and 2, words and strings of hashes in Experiment 3, and dotted lines in Experiment 4) rules out an interpretation in terms of target undershooting (see Radach et al.'s, 1995, low-level hypothesis). The participants did not undershoot when targeting leftpresented words. If it is assumed that a single mechanism was at work for both presentation sides, this means that the undershooting hypothesis can be ruled out for right targeting as well (but see Radach \& McConkie, 1998). More generally, the finding that participants apparently used different inspection strategies, depending on the type of stimulus they were targeting (discrete vs. continuous), suggests that the off-centeredness observed for discrete stimuli did not result only from oculomotor constraints. This effect was not due to an erroneous perceptual assessment of the middle of the stimulus either. In none of the bisection experiments reported here did the initial fixation position exactly coincide with the subjective midpoint eventually selected by the participants. Typically, the participants landed close to the center of a left-presented discrete stimulus ( \pm 0.3 character positions) and left of the midpoint of a right-presented discrete stimulus $(=-1.2$ character positions). They subsequently made a rightgoing saccade toward the subjective midpoint before pressing the button. This suggests that they probably knew that they were not landing in the middle of right-presented stimuli.
What about the role of lexical constraints in determining landing positions in words (see Radach et al.'s, 1995, processing adaptation hypothesis)? In favor of this hypothesis, remember that a leftward bias was observed for both left and right presentations in Experiment 1. Moreover, this effect was more pronounced in Experiment 1that is, when the task required lexical processing - than in the other experiments, in which only perceptual processing of the stimulus was presumably required. This suggests that the participants targeted the word's beginning in order to be in the location where lexical information can usually be found. The analysis of saccade latency did not support this view, however. As was suggested by Radach et al., instantiating such a high-level strategy presumably takes time, so relatively longer initial saccade latencies were expected in Experiment 1 (lexical decision task) than in the other experiments (bisection task). Moreover, longer saccade latencies were expected for words, as compared with solid lines (unlike words, solid lines did not elicit any left/right asymmetry). Contrary to these predictions, similar saccade latencies were found on linguistic stimuli in all the experiments. A significant difference was found between words and nonlinguistic stimuli in Experiments 2 and 3. However, the effect went in the wrong direction, with shorter not longer saccade latencies for words than for nonlinguistic stimuli (for a similar result, see Rayner \& Fischer, 1996, and Vitu et al., 1995). Targeting the beginning of a word in Experiment 1 actually consumed less time than targeting the middle of a solid line in Experiments 2, 3, and 4 .

There are some other aspects of our results that also argue against the idea that lexical constraints can (or can alone) explain the observed asymmetry. For example, the fact that the bisection task elicited comparable landing position patterns for words (either high or low frequency), nonwords (either regular or irregular), and nonlinguistic stimuli, such as strings of hashes and dotted lines, suggests that some other factors must have taken effect. The results for the location of the subjective midpoint are also at odds with the role of lexical constraints as an important determinant of landing position. Unlike Fischer (1996), we did not find any differences between the bias observed on words, nonwords, strings of hashes, dotted lines, and solid lines. These results seem inconsistent with Fischer's (2000) hypothesis that lexical access involves attentional focusing on the initial letters of a word (in order to establish a cohort of potential matches with entries in the mental lexicon) and that this strategy would yield overrepresentation of the word's beginning, resulting in a leftwardbiased subjective midpoint. More generally, the present data seem to rule out a high-level explanation of eye guidance during reading in terms of computing some sort of optimal position based on knowledge of the language. If lexical factors had no effect here, with tasks in which eye guidance could more plausibly be directed by higher level and/or strategic factors, they are unlikely to guide eye movements in natural reading. 
If neither low-level oculomotor factors nor high-level linguistic factors can account for the obtained landing position asymmetry, then what can? Purely perceptual factors are not likely to account for our data either. Clearly, an explanation in terms of mere letter visibility is not sufficient (i.e., the fact that visual acuity rapidly declines between the center of the retina and the periphery; Jacobs, 1979). Since visual acuity declines symmetrically on either side of the fixation center, both the OVP and the PLP should be located at the word's midpoint. The OVP effect has sometimes been seen as a consequence of hemispheric specialization(Brysbaert \& d'Ydewalle, 1988; Brysbaert, Vitu, \& Schroyens, 1996). In this view, visual word recognition is achieved by neural mechanisms situated in the left hemisphere of the brain, so a word presented in the left visual field suffers from a processing delay equal to the time required to transmit information from the right to the left hemisphere. However, this interpretation was challenged by the finding that both the OVP and the PLP tended to be to the right of center for languages read from right to left (Deutsch \& Rayner, 1999). The results of the present study extended Deutsch and Rayner's by showing that landing positions depend on the language used to train bilinguals prior to the experiment. When the participants read a French text beforehand, the landing pattern on discrete stimuli was asymmetrical, like the one obtained for French monolinguals. But when right-to-left exploration was induced, the asymmetry disappeared. These results clearly rule out hemispheric asymmetry as the sole determinant of landing position in this type of task.

Alternatively, the off-center position of the OVP could be linked to reading habits that govern sensory information processing (Farid \& Grainger, 1996). It is well known that the useful visual field is asymmetrical in reading, extending farther to the right for left-to-right languages such as English and French and farther to the left for right-toleft languages such as Hebrew (Pollatsek, Bolozky, Well, $\&$ Rayner, 1981; Rayner, 1998). This is clearly relevant to the present discussion. If letters presented, say, on the right are perceived better than those presented on the left (which was presumably the case for our French monolingual participants), landing to the left of the middle makes sense, since this is likely to enhance overall letter visibility (Nazir, O'Regan, \& Jacobs, 1991). Note, however, that the asymmetry observed in the present study was not restricted to linguistic stimuli, and one can wonder why reading habits would enter into play for stimuli like strings of hashes or dotted lines. What is more, these nonlinguistic stimuli were not presented in a reading context (except in Experiments 5 and 6, in which a short reading session was proposed to the participants prior to the bisection task). Apparently, the asymmetry of the visual field is not restricted to reading situations. This asymmetry seems to be dependent on some low-level property of the stimuli being processed, like the presence of spaces between discrete elements (words, nonwords, strings of hashes, and dotted lines all shared this property, unlike solid lines).
Why should the asymmetry of the visual field be restricted to discrete stimuli? A tentative explanation that seems consistent with the results of Experiment 5 is that the PLP effect for isolated stimuli is due to attentional processes (associated with reading habits) that develop for any type of discrete stimulus, whether or not reading is actually required.

Experiment 6 addressed the question as to whether the landing position asymmetry can be modulated by any task involving right-left scanning. French monolinguals were asked to perform a short preliminary task in which they had to move their eyes from right to left (i.e., in the direction opposite to their reading habits). In this situation, the left/right asymmetry for discrete stimuli was eliminated. As in Experiment 5, the mechanisms of the influence exerted by the training phase remain difficult to untangle here (facilitatory or inhibitory effects of oculomotor programming and/or attention allocation). We are inclined to favor the interpretation in terms of attentional scanning, mentioned above. For example, it could be argued that, in all of the conditions in which reading habits were not experimentally disrupted, the participants, whether bilinguals or monolinguals, anticipated doing left-to-right attentional scanning of discrete stimuli and took the direction of this upcoming attentional scanning into account when computing the incoming saccade. In other words, they tried to land left of center so as to be able to explore the stimulus in the left-to-right direction, thus left-shifting PLP for both left and right presentations (again, we are assuming here that our French/Arabic bilinguals were more accustomed to reading from left to right than from right to left). True, as far as left presentation is concerned, a leftof-center position was clearly obtained only in Experiment 1 (lexical decision task). In the subsequent experiments, saccades directed toward left-presented discrete stimuli actually landed close to the center (although an asymmetry was still obtained relative to the right-presentation condition). Note, however, that in these experiments, the task was explicitly to reach the center of the stimulus. Landing left of center would thus have involved programming two successive saccades in opposite directions. The fact that the PLP was clearly left of center for right presentation, despite the requirements of the task, suggests that landing left of center probably provided some processing advantage for the bisection task as well. For this reason, it may be important to note that the direction of the final adjustment (if any) after landing in a discrete stimulus was usually in the same (right-going) direction, regardless of the direction of the entry saccade.

To conclude, we believe that our results support the idea that the landing position in this study was determined by an eye-guiding mechanism based on a perceptual lowlevel preprocessing step that detected the presence or absence of spaces between characters. When the stimulus turned out to be discrete, the participants took the direction of visual exploration into account and attempted to land left of center (for a left-to-right language), in prepa- 
ration for subsequent left-to-right attentional scanning. When the stimulus turned out to be continuous, no attentional scanning was implemented, and the landing position pattern was symmetrical.

\section{REFERENCES}

Broerse, A. C., \& ZwaAn, E. J. (1966). The information value of initial letters in the identification of words. Journal of Verbal Learning \& Verbal Behavior, 5, 441-446.

Brysbaert, M., \& D'Ydewalle, G. (1988). Callosal transmission in reading. In G. Lüer, U. Lass, \& J. Shallo-Hoffman (Eds.), Eye movement research: Physiological and psychological aspects (pp. 246266). Göttingen, The Netherlands: Hogrefe.

Brysbaert, M., Vitu, F., \& Schroyens, W. (1996). The right visual field advantage and the optimal viewing position: On the relation between foveal and parafoveal word recognition. Neuropsychology, 18, 385-395.

Chorron, S., \& DE Agostini, M. (1995). Reading habits and line bisection: A developmental approach. Cognitive Brain Research, 3, 5158 .

Chokron, S., \& Imbert, M. (1993). Influence des habitudes de lecture sur la perception de l'espace [Influence of reading habits on space perception]. Images et langages (Actes du Colloque CNRS, pp. 47-63). Paris: Comité National de la Recherche Scientifique.

Cö̈FfÉ, C., \& O'Regan, J. K. (1987). Reducing the influence of nontarget stimuli on saccade accuracy: Predictability and latency effects. Vision Research, 27, 227-240.

Deutsch, A., \& RAYNer, K. (1999). Initial fixation location effects in reading Hebrew words. Language \& Cognitive Processes, 14, 393-421.

FARID, M., \& GRAINGER, J. (1996). How initial fixation position influences visual word recognition: A comparison of French and Arabic. Brain \& Language, 53, 681-690.

FINDLAY, J. M. (1982). Global visual processing for saccadic eye movements. Vision Research, 22, 1033-1045.

FischeR, M. H. (1996). Bisection performance indicates spatial word representation. Cognitive Brain Research, 4, 163-170.

Fischer, M. H. (1999). An investigation of attention allocation during sequential eye movement tasks. Quarterly Journal of Experimental Psychology, 52A, 649-677.

Fischer, M. H. (2000). Word center is misperceived. Perception, 29, 337-354.

Henderson, J. M., \& Ferreira, F. (1990). Effects of foveal processing difficulty on the perceptual span in reading: Implications for attention and eye movement control. Journal of Experimental Psychology: Learning, Memory, \& Cognition, 16, 417-429.

HyönÄ, J., \& Pollatsek, A. (1998). Reading Finnish compound words: Eye fixations are affected by component morphemes. Journal of Experimental Psychology: Human Perception \& Performance, 24, 1612-1627.

HyönÄ, J., \& Pollatsek, A. (2000). Processing of Finnish compound words in reading. In A. Kennedy, R. Radach, D. Heller, \& J. Pynte (Eds.), Reading as a perceptual process (pp. 65-87). Amsterdam: Elsevier.

InHoff, A. W. (1989). Parafoveal processing of words and saccade computation during eye fixations in reading. Journal of Experimental Psychology: Human Perception \& Performance, 15, 544-555.

Inhoff, A. W., Pollatsek, A., Posner, M. I., \& Rayner, K. (1989). Covert attention and eye movements during reading. Quarterly Journal of Experimental Psychology, 41A, 63-89.

JACOBS, R. J. (1979). Visual resolution and contour interaction in the fovea and periphery. Vision Research, 19, 1187-1196.

McConkie, G. W., Kerr, P. W., Reddix, M. D., \& Zola, D. (1988). Eye movement control during reading: The location of initial eye fixations on words. Vision Research, 27, 227-240.

McConkie, G. W., Kerr,P. W., Reddix, M. D., Zola, D., \& Jacobs, A. M. (1989). Eye movement control during reading: II. Frequency of refixating a word. Perception \& Psychophysics, 46, 245-253.

Morris, R. K., Rayner, K., \& Pollatsek, A. (1990). Eye movement guidance in reading: The role of parafoveal letter and space information. Journal of Experimental Psychology: Human Perception \& Performance, 16, 268-281.
Nazir, T. A., O'Regan, J. K., \& Jacobs, A. M. (1991). On words and their letters. Bulletin of the Psychonomic Society, 29, 171-174.

O'Regan, J. K. (1981). The "convenient viewing position" hypothesis. In D. F. Fisher, R. A. Monty, \& J. W. Senders (Eds.), Eye movements: Cognition and visual perception (pp. 289-298). Hillsdale, NJ: Erlbaum.

O'Regan, J. K., \& JACoBS, A. M. (1992). Optimal viewing position effect in word recognition: A challenge to current theory. Journal of Experimental Psychology: Human Perception \& Performance, 18, 185197.

O’Regan, J. K., \& Levy-Schoen, A. (1987). Eye movement strategy and tactics in word recognition and reading. In M. Coltheart (Ed.), Attention and performance XII: The psychology of reading (pp. 263284). Hillsdale, NJ: Erlbaum.

O’Regan, J. K., Levy-Schoen, A., Pynte, J., \& Brugaillère, B. (1984). Convenient fixation location within isolated words of different length and structure. Journal of Experimental Psychology: Human Perception \& Performance, 10, 250-257.

Pollatsek, A., Bolozky, S., Well, A. D., \& Rayner, K. (1981). Asymmetries in the perceptual span for Israeli readers. Brain \& Language, 14, 174-180.

RADACH, R, \& Kempe, V. (1993). An individual analysis of initial fixation positions in reading. In G. d'Ydewalle \& J. Van Rensbergen (Eds.), Perception and cognition: Advances in eye movement research (pp. 213-225). Amsterdam: North-Holland.

Radach, R., Krummenacher, J., Heller, D., \& Hofmeister, J. (1995). Individual eye movement patterns in word recognition: Perceptual and linguistic factors. In J. M. Findlay, R. Walker, \& R. W. Kentridge (Eds.), Eye movement research: Mechanisms, processes and applications (pp. 421-432). Amsterdam: Elsevier.

RADACH, R. \& MCCONKIE, G. W. (1998). Determinants of fixation positions in words during reading. In G. Underwood (Ed.), Eye guidance in reading and scene perception (pp. 77-100). New York: Elsevier.

RAYNER, K. (1975). The perceptual span and peripheral cues in reading. Cognitive Psychology, 7, 65-81.

RAYNER, K. (1979). Eye guidance in reading: Fixation location within words. Perception, 8, 21-30.

RAYNER, K. (1986). Eye movements and the perceptual span in beginning and skilled readers. Journal of Experimental Child Psychology, 41, 211-236.

RAYNER, K. (1998). Eye movements in reading and information processing: 20 years of research. Psychological Bulletin, 124, 372-422.

RAYNER, K., \& Fischer, M. H. (1996). Mindless reading revisited: Eye movements during reading and scanning are different. Perception \& Psychophysics, 58, 734-747.

Rayner, K., Fischer, M. H., \& Pollatsek, A. (1998). Unspaced text interferes with both word identification and eye movement control. Vision Research, 38, 1129-1144.

RAYNER, K., \& MORRIS, R. K. (1992). Eye movement control in reading: Evidence against semantic preprocessing. Journal of Experimental Psychology: Human Perception \& Performance, 18, 163-172.

Rayner, K., Sereno, S. C., \& Raney, G. E. (1996). Eye movement control in reading: A comparison of two types of models. Journal of Experimental Psychology: Human Perception \& Performance, 22, 11881200.

Rayner, K., Well, A. D., Pollatsek, A., \& Bertera, J. H. (1982). The availability of useful information to the right of fixation in reading. Perception \& Psychophysics, 31, 537-550.

Trésor de la langue française [French language frequency counts] (1971). Nancy, France: Centre National de la Recherche Scientifique.

Underwood, G., Clews, S., \& Everatt, J. (1990). How do readers know where to look next? Local information distributions influence eye fixations. Quarterly Journal of Experimental Psychology, 42A, $39-65$.

Vitu, F., O'Regan, J. K., Inhoff, A. W., \& Topolski, R. (1995). Mindless reading: Eye-movement characteristics are similar in scanning letter strings and reading texts. Perception \& Psychophysics, 57, 352364.

Vitu, F., O’Regan, J. K., \& Mittau, M. (1990). Optimal landing position in reading isolated words and continuous text. Perception \& Psychophysics, 47, 583-600. 


\section{DUCROT AND PYNTE}

\section{NOTES}

1. O'Regan (1981) originally used the expression convenient viewing position, rather than optimal viewing position, to refer to this phenomenon.

2. As Alexander Pollatsek brought to our attention, Radach et al.'s (1995) low-level hypothesis is contingent on the theory of noise one adopts.

3. Note that the position the participants eventually selected as the subjective midpoint was not the true midpoint either. It could be that there is an illusion related to attention, so that readers are attending to a point other than the one they are fixating but think they are fixating their attention center. If this is true, the results reported here concerning the subjective midpoint must be treated with caution. We are grateful to Alexander Pollatsek for pointing out this idea.

4. In Experiments 5 and 6, the participants had to move their eyes from left to right (or from right to left) during both the calibration and the trai ning phases. This probably provided some additional training.

(Manuscript received March 9, 1999;

revision accepted for publication January 30, 2002.) 